\title{
Entre ciel et terre: mutation de l'expérience à propos de localisation géographique à l'ère connectique
}

\author{
CHENG YU PAN
}

Doutor em Artes Plásticas e Ciências da Arte, Paris 1 Panthéon-Sorbonne. Nascido em Taipei (Taiwan), Cheng Yu Pan vive e trabalha em Paris e Limoges. Artista e pesquisador, ele investiga as questões relacionadas aos fenômenos da rede e da conectividade e seus impactos culturais. Entre suas formas de expressão artística predominam a instalação de dispositivos de net.art e vídeo arte. E-mail: noixdarec@gmail.com 


\section{- RÉSUMÉ}

Les réseaux sont aujourd'hui un territoire à la fois riche et mythique pour la civilisation humaine. Riche, car la puissance du virtuel qu'ils portent atteint un degré sans précédent, mythique parce que nous ne connaissons pas encore clairement son effet à long terme sur nous. Je m'intéresse donc aux arts en réseaux et aux phénomènes gravitant autour de ce sujet, particulièrement au devenir de la perception que nos civilisations ont du monde. Quand je pratique une activité artistique, je souhaite qu'elle consiste à fabriquer des «phénomènes » non-ordinaires dans le monde ordinaire. Mes travaux plastiques portent souvent sur l'installation, la photographie et la vidéo, toutes ces formes se lient d'une manière ou d'une autre aux réseaux. Trois facteurs - le « regard », l'« écran », et la " connectivité »- me sont devenus essentiels, et constituent la problématique principale de mes créations. Dans cet article, je proposerai d'abord une interrogation sur l'expérience de l'utilisateur des réseaux - de la télécommunication à la navigation sur les services géographiques, ces expériences refléteraient le devenir de l'état d'existence de l'individu. En suite, deux de mes travaux plastiques seront présentés, qui permettront d'illustrer les conceptions que je possède pour la mise en œuvre de mes créations : l'« eyeTunnel » s'agit d'une vidéo représentant une mise en abyme des portraits sous la forme d'écrans, qui se pénètrent au travers de leurs pupilles; et le projet «iTunnel » vise à construire un tunnel virtuel par le biais des dispositifs et protocoles informatiques.

\section{- MOTS-CLÉS}

Net.art, GPS Art, regard, connectivité, virtualité.

\section{- RESUMO}

Atualmente as redes apresentam um território ao mesmo tempo rico e mítico para a civilização humana. Rico, pelo potencial do virtual que chegou a uma altura sem precedentes, e, mítico, pela razão que nos não conhecemos ainda seus efeitos sobre nós a longo prazo. Meu interesse concentra-se na arte em rede, nos fenômenos que gravitam em torno deste tema, e sobretudo na percepção do mundo pela nossa civilização numa perspectiva do futuro. Quando realizo uma ação artística, meu objetivo é de fabricar fenômenos incomuns no mundo comum. Meus trabalhos plásticos usam frequentemente a instalação, a fotografia, e o vídeo, sempre ligados de alguma forma às redes. Três fatores - o olhar, a tela, e a conectividade - tornaram-se essenciais e constituem a problemática principal das minhas criações. Neste artigo, proponho inicialmente investigar a experiência do usuário de redes: das telecomunicações à navegação nos serviços geográficos (geoprocessamento); essas experiências refletem o estado de existência do indivíduo. Como resultado, apresento dois trabalhos artísticos nos quais desenvolvo a problemática em questão: o "eyeTunnel" é um vídeo que mostra uma sequência de retratos conectados pelo olhar encadeando as pupilas dos olhos das pessoas retratadas, e o projeto "iTunnel" visa a construção de um túnel virtual através de dispositivos e protocolos informáticos.

\section{PALAVRAS-CHAVE}

Net arte, GPS arte, olhar, conectividade, virtualidade.

\section{Perspective du lieu et de la frontière, l'absence de l'utilisateur}

Paul Virilio a remarqué que l'influence de la révolution des transports sur l'architecture et l'urbanisme des mégapoles a pour conséquence le déplacement du paradigme entrée/sortie. La force motrice des animaux de traction a déplacé l'entrée/ sortie des frontières nationales jusqu'à l'enceinte des villes ; la force de propulsion mécanique l'a encore déplacée jusqu'aux gares et stations de bus, puis aux garages individuels. Enfin, la révolution des réseaux informatiques a déplacé ce paradigme 
jusqu'au modem du PC (personal computer, ordinateur individuel) qui assure l'accès aux réseaux. Si l'idée du modem n'est pas tellement compréhensible, prenons un terme plutôt visuel et disons que : ce paradigme, c'est-à-dire la frontière entre l'individu et le monde qui l'environnent s'est aujourd'hui rétracté en une surface, celle de l'écran d'ordinateur placé devant lui.

Dans ce contexte, l'idée de sortir ne signifie plus franchir le seuil de chez soi pour marcher ou prendre un quelconque moyen de transport afin de rejoindre sa destination. Traditionnellement, la carte géographique permet de se retrouver, se positionner dans son environnement, aujourd'hui, mise en réseau, elle sert le plus souvent à la découverte de nouveaux lieux, sans avoir à sortir de chez soi.

Par conséquent, parler de l'absence de soi n'est pas obligatoirement lié au fait d'une absence physique. On peut être absent simplement en raison du fait qu'on est connecté aux réseaux. Marshall McLuhan emploie le terme de discarnate man (l'homme désincarné) pour décrire l'état d'une personne qui utilise le médium. « Le médium électronique nous transporte instantanément à n'importe quel endroit où on veut y aller. Quand nous téléphonons, [...] nous sommes là-bas et ils sont ici. " ${ }^{1}$ En un certain sens, lorsqu'on est au téléphone, notre attention s'évade provisoirement du contexte dans lequel nous communiquons. II s'absente. Mais où est-t-il ? Est-il vrai que « nous sommes là-bas et ils sont ici », comme dit McLuhan ? Quand je téléphone à Uberlândia, par exemple, je sens que je suis avec mon interlocuteur, je ne me sens pourtant pas présent là-bas. Prenons un exemple plus à la mode : quand je parle avec un ami à Taïwan via Skype avec la webcam, je ne me sens pas à Taïwan. Pourtant, je sens que je suis devant lui. Que se passe-t-il alors dans ces communications?

D'abord, il semble que nous sommes en fait retirés du monde ordinaire et nos esprits, portés par les voix vives et/ou les images en temps réel qui nous occupent lors des échanges, circulent dans l'univers virtuel, là où l'idée de distance et de position physique n'a pas de sens. Nous ne sommes nulle part, plutôt qu'ubiquistes, au moment d'échanger en réseau. Ensuite, notons la sensation d'être avec ou devant notre interlocuteur. Cela monte le fait que lors de ces communications, nous nous situons quant même dans un espace, quoi qu'il soit tellement abstrait que ne corresponde nulle part du monde ordinaire. Cet espace est pour moi l'essentiel de ce que l'on appelle cyberespace, fameux terme mystérieux qu'il serait réducteur de n'associer qu'aux espaces visuels en 3D.

\section{Expérience sur le SIG : un voyage astral}

Cette expérience sur le réseau téléphonique est plutôt abstraite. Trente ans après McLuhan, nous bénéficions de réseaux informatiques portant une énorme puissance du virtuel, dont les systèmes d'information géographique (SIG²) comme Google Maps, Google Earth ou encore Maps de la compagnie Apple, étalent devant nous chaque recoin du monde. Un des nouveaux effets de Maps annoncés par Apple en

\footnotetext{
«Electric media transport us instantly wherever we choose. When we are on the phone [...] we are there and they are here. " Marshall MCLUHAN, écrits assemblés dans. Essential McLuhan, Eds. E. McLuhan and F. Zingrone, London: Routledge, 1995. p. 289.

2 Système d'information géographique (en Anglais : Geographic information system, GIS), un système d'information permettant d'organiser et de présenter des données alphanumériques spatialement référencées, ainsi que de produire des plans et des cartes. Ses usages couvrent les activités géomatiques de traitement et diffusion de l'information géographique.
} 
2012 s'appelle «Flyover». Comme son nom l'indique, il s'agit d'une expérience consistant à survoler la Terre, similaire au simulateur de vol (flight simulator) dans Google Earth.

L'utilisation de ces SIG n'est donc pas si abstraite par rapport à celle du téléphone; l'expérience serait plus visuelle mais en même temps, bien plus compliquée. À part le phénomène de l'absence qui est sûrement l'un des facteurs capitaux, l'effet de survoler en serait une impression populaire, mais celle-ci ne semble pas pouvoir illustrer ce genre d'expérience de façon profonde nous permettant une compréhension essentielle. Le terme voyage astral pourrait être approprié à ce propos. Selon wikipédia, le voyage astral concerne « l'impression que l'esprit se dissocie du corps physique pour vivre une existence autonome et explorer librement l'espace environnant. » La première partie - se dissocier du corps physique - correspondrait bien à l'absence de l'utilisateur, quant à l'autre partie - explorer librement l'espace environnant - ne se souvient pas-t-on d'avoir survolé l'emplacement de chez soi dans Google Earth et s'être écrié :»Ah voilà ! Là, c'est chez nous»? Le SIG est comme une «sorcière» informatique, une secte contemporaine nous offrant l'expérience de l'autoscopie, celle-ci à une échelle mondiale.

M'apercevant du fait que la frontière entre l'individu et le monde s'est modifiée avec l'apparition de l'écran placé entre eux, et que l'idée de la position et de la distance semble s'être modifiée à travers les voyages astraux, je choisis d'élaborer deux actes paradoxaux, dont l'un est à l'opposé de ces résultats merveilleux menés par la technologie. Je propose de marcher dans la perspective de retrouver la distance physique oubliée dans la connexion des réseaux (iTunnel 2012); tout en associant le fait de creuser un tunnel virtuel en empruntant la puissance de la technologie (eyeTunnel, iTunnel 2006, iTunnel 2012).

\section{TRAVAUX PLASTIQUES}

\section{1. eyeTunnel Vidéo, couleur, muet, 2»22', 2010}

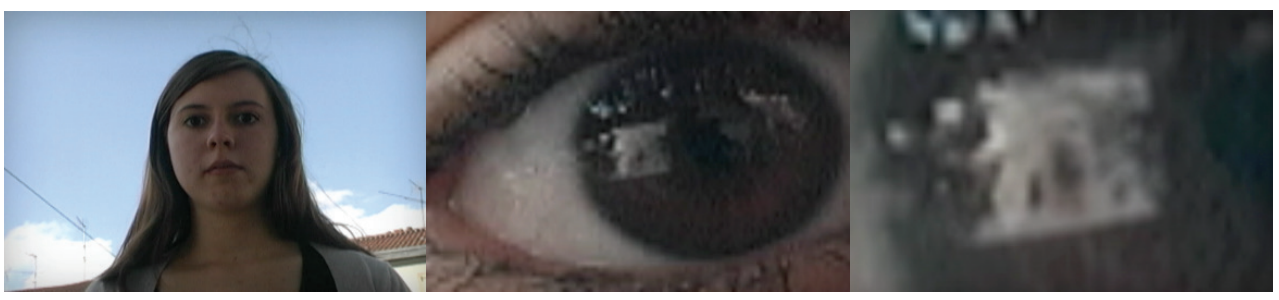

Figure 1 - eyeTunnel, vidéo, couleur, muet, 2"22', Cheng Yu PAN, 2010, courtesy de l'artiste.

Cette vidéo représente une mise en abyme des portraits de mes amis, sous la forme d'écrans, qui se pénètrent, les uns les autres au travers de leurs pupilles. II s'agit d'un enchaînement de regards, réalisé en écho à l'utilisation intensive des dispositifs photographiques mis en ligne par les utilisateurs, par le biais des échanges d'informations instantanés des réseaux informatiques.

Lors du tournage, j'ai demandé ces personnes de s'efforcer de ne pas cligner les yeux (ce qui paraît malaisé pour la plupart des gens). La raison de cette sollicitation résulte de la volonté de transformer la pupille - qui est à l'origine un organe - en un médium, c'est-à-dire, quand la subjectivité et l'objectivité des personnes ne cessent de varier à cause du fait de regarder et d'être regardé, la pupille deviendra l'issue 
des regards, le passage de ces échanges de subjectivité et objectivité. En un mot, la pupille est mon tunnel.

\section{a. Enjeux}

La première idée de ce projet est née en 2006, au moment où les services de relations interpersonnelles comme Facebook venaient d'apparaître en ligne. Ce projet a connu plusieurs rectifications conceptuelles et techniques avant de revêtir sa forme actuelle. Au début, j'ai demandé à des amis qui se situaient en différents endroits du monde de réaliser leurs autoportraits avec leur appareil numérique et de me les envoyer. À partir de ces photographies, je souhaitais ensuite fabriquer une vidéo interactive en ligne à l'aide de la technologie Flash.

Si cette première idée avait pu être réalisée, ce projet aurait pu être rangé sans aucun doute dans l'art.net (art en réseaux). En raison de considérations techniques et esthétiques, j'ai décidé de ne pas me servir de ces photos et de tourner moi-même des portraits de mes amis en vidéo. La question s'est posée : au niveau de la forme, ce travail relève évidement de l'art-vidéo. Or, compte tenu de mon intention, de la conception et du contenu de ce projet, devait-il être considéré encore comme un net. art ? Dans cette vidéo, je perçois la formation d'un réseau qui s'est formé, et ce réseau s'inscrit toujours en écho avec les réseaux actuels. II s'agirait donc la question de la définition de l'art.net. Je n'en ai pas personnellement encore de réponse précise, c'est pourquoi l'avis d'autrui me sera fort précieux.

\section{2. iTunnel}

Si eyeTunnel consiste en un tunnel virtuel sur lequel l'aspect spirituel s'avère prononcé - puisqu'il est construit à travers les regards des personnes, leurs pupilles, le second projet iTunnel viserait plutôt à mettre en évidence la puissance de la visualisation fournie par les nouvelles technologies et largement appréhendée par la connectivité des réseaux.

Ce projet a eu deux versions dans la pratique fortes différentes : en 2006, il s'agit d'une installation s'efforçant de construire un tunnel visuel à travers des dispositifs liés aux réseaux. Invité par la galerie LaVitrine à Limoges en début 2012 pour la troisième édition de Consommé d'Artiste, ce projet a évolué pour aboutir vers une seconde version : iTunnel - Trou de ver. II est ainsi devenu plus complexe en combinant à la fois de la performance, le Net.art et Map Art.

\section{Premiere version : Itunnel 2006}

Installation, févier 2006, Paris.

La première version de ce projet fut conçue en 2006, et les concepts principaux basés sur ma déduction concernant les dispositifs technologiques et les réseaux ont été ainsi définis : si les dispositifs de visualisation tels que les écrans présentent une certaine tendance, celle-ci pourrait être de disparaître, dans la mesure où leur volume ne cesse de diminuer, tandis que s'accroît leur maniabilité. Sachant que la valeur d'un outil ne réside pas dans sa forme, mais dans sa fonctionnalité, l'idéal consisterait alors à garder cette dernière et éliminer la première. Un des exemples pourrait être le suivant : les lentilles de 
contact. En ce qui concerne les réseaux, sa caractéristique la plus importante me semble être la pénétrabilité induite par la connectivité, et grâce à cette dernière, les réseaux paraissent alors être en mesure de pénétrer l'ensemble des obstacles physiques.

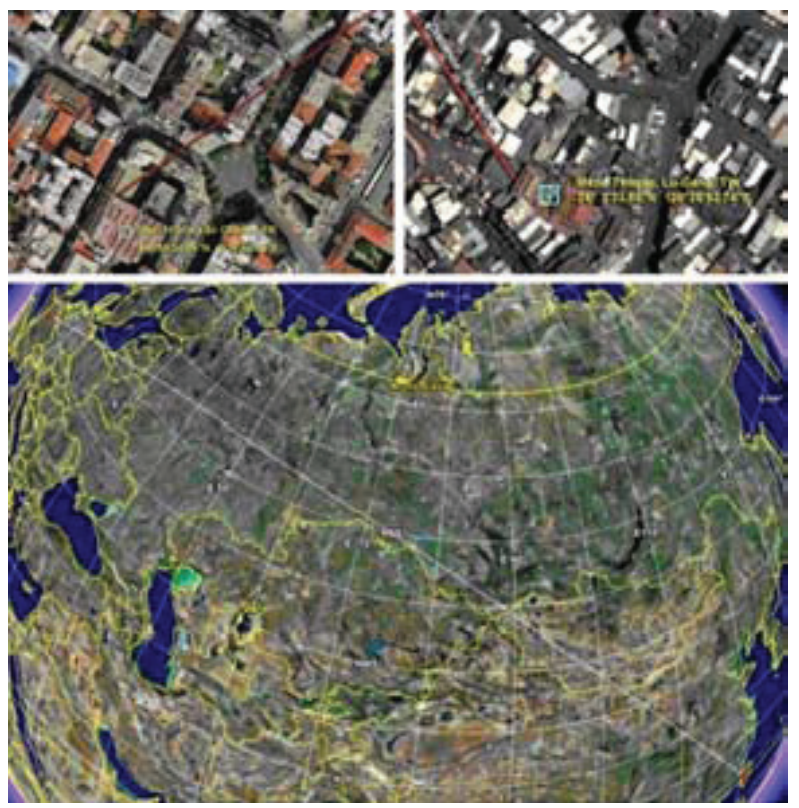

Figure 2 - iTunnel, affiche plan Saint Charles, Cheng Yu PAN, 2006, courtesy de l'artiste.

Dans le cadre de ce projet, j'ai posé un écran sur le mur, alors qu'une webcam a été accrochée sur le verso de ce même mur, les deux se trouvant reliés à l'intranet (réseau interne). Cette installation simple simule le percement d'un mur. On pourrait avancer que l'écran, ainsi que la zone du mur couvert par celui-ci sont dématérialisés. J'ai ainsi pénétré une série de murs dans un bâtiment à l'aide de plusieurs écrans reliés par ordinateurs et webcams. L'ensemble se trouve à l'origine d'un tunnel.

Au dernier écran accroché sur le dernier mur du bâtiment, j'ai enfin mis en réseaux les images d'une webcam, prolongeant ainsi le tunnel de 8000 kilomètres joignant Paris et Taïwan.

Le principe de ce projet est simple : grâce à une caméra installée sur un côté d'un mur et d'un moniteur sur l'autre côté, on obtiendra un effet de pénétration. À l'aide de la transmission de données entre la caméra et l'écran se déroulant par le réseau (l'Intranet $\left.{ }^{3}\right)$, on pourra donc pénétrer tous les murs/obstacles. Plus encore, avec la connexion Internet, il deviendra, pour nous, possible de pénétrer partout, en traversant les montagnes, les rivières... et la Terre elle-même!...

\section{b. Conformations}

Supports techniques: Caméras IP sans fil, routeur sans fil, écrans plats LCD avec ordinateurs PC ou, ordinateurs portables, webcam installée à distance (Taïwan)

\footnotetext{
3 Intranet : un réseau informatique utilisé à l'intérieur d'une entreprise ou de toute autre entité organisationnelle utilisant les mêmes techniques et protocoles de communication d'Internet (IP).
} 


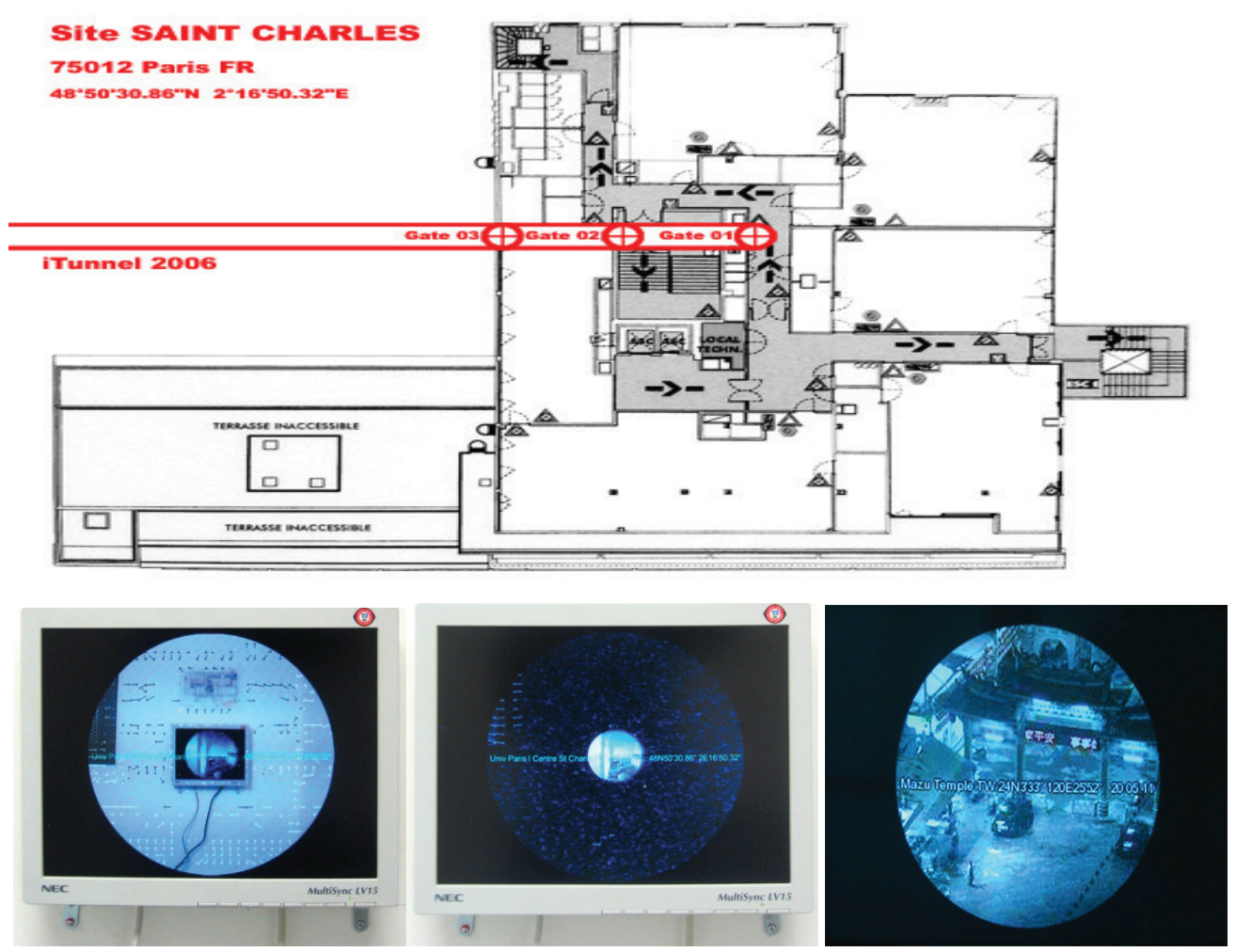

Figure 3 - iTunnel, installation des écrans plats pour, Cheng Yu PAN, 2006, courtesy de l'artiste.

\section{Deuxième version : iTunnel 2012 - Trou de ver}

Installation multimédia et performance en forme de tracés GPS en temps réel, photographies numériques retouchées et mises en lignes.

Page Web: http://consommedartiste.hyperreel.net/?p=1065

Site de l'exposition «Consommé d'Artiste IIl» : http://consommedartiste.hyperreel.net

\section{c. Élaboration}

Ce projet a été réalisé et présenté dans l'exposition intitulée Consommé d'Artiste III, organisée par Kristina Depaulie dans la galerie LaVitrine à Limoges, en France en février 2012. Le fait que ce projet soit fortement intégré dans le cadre de l'exposition, rend nécessaire de s'attarder d'abord sur cette dernière. Cette exposition qui présente principalement des performances, parmi d'autres, s'avère plutôt expérimentale et ce qui me paraît particulier, c'est que les artistes invités se situent en différents endroits du monde (France, Espagne, Grèce, Chine...) et la plupart d'entre eux (moi inclus) ne sont pas présents physiquement dans la galerie. Les artistes sont conviés à réaliser leurs performances localement avant d'envoyer des traces - qu'elles soient documentées ou en temps réel - à la galerie. Durant trois semaines d'exposition, la galerie organise trois vidéoconférences permettant au public de discuter avec chaque artiste via Skype. Ainsi, Consommé d'Artiste III a pu être célébré autour d'une délicieuse soupe préparée par la curatrice. 


\section{d. Conception}

Le concept de trou de ver proposé par Einstein et Rosen suggère l'idée d'un tunnel provoqué par les trous noirs qui pénétrerait l'espace-temps et qui permettrait de voyager entre deux lieux extrêmement éloignés. Si ce terme demeure aujourd'hui purement théorique dans le champ scientifique, il se trouve pourtant déjà réalisé, au niveau visuel, à travers les réseaux. Au moment de la connexion, la rencontre s'opère par le biais des outils de communication numériques (Skype), grâce auxquels on arrive chez notre interlocuteur. Les espaces physiques entre ces deux lieux disparaissent et l'écran n'est autre qu'un trou de ver numérique.

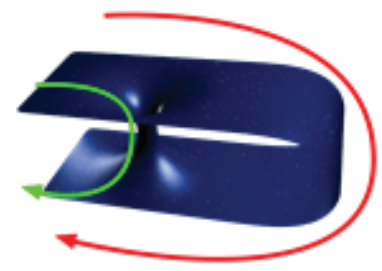

Figure 4 - Shéma du trou de ver proposé par Einstein et Rosen.

Cette disparition de l'espace physique entre deux nœuds interférant dans le réseau me fascine particulièrement. II s'agit d'une action/performance, ou plus précisément d'un voyage nécessaire pour retrouver cet espace perdu. L'ensemble du projet réalisé est présenté sur le site Web de l'exposition, sur lequel les tracés GPS de mon déplacement sont affichés en temps réel, accompagnés des photographies prises pendant les trajets - par le biais de Photoshop. La superposition de ces photos se trouve appréhendée sous la forme d'un point rond, un trou/tunnel visuel.

\section{e. Conformations}

\section{Destination}

Le premier pas consiste à déterminer la destination du tunnel. Cela permettra ensuite de déterminer sa direction. Puisque la puissance de la pénétration du système numérique se révèle suffisamment importante pour traverser le noyau terrestre, il ne me semble pas indispensable de limiter la sphère de tunnel à un seul pays. S'il existait un moyen susceptible d'offrir un accès immédiat aux endroits où je souhaiterais m'y rendre en un clin d'œil, par la nature humaine. Je désirerais tout d'abord retourner au lieu d'où je viens et effectuer une rétrospective. Mon choix de destination a porté tout naturellement sur Taïwan. Seulement, le site précis n'est pas mon propre domicile, mais les ruines de Ketangalan qui représentent, pour moi, mon authentique chez moi dans un sens plus profond et plus large.

Au cours de mes études universitaires, j'ai découvert mes origines aborigènes. Jusqu'à ce moment-là, je croyais fermement que mes ancêtres avaient immigré il y a quatre cents ans depuis la Chine continentale. Nos aînés ne nous ont jamais parlé de cette histoire, car les Aborigènes ont toujours été traités de façon discriminatoire.

Suivant mon habitude, l'idéalité de l'œuvre s'avère pour moi perpétuellement prioritaire par rapport à la faisabilité de l'œuvre (bien que la faisabilité de la mise en œuvre, ainsi qu'une variété de détails soient également non-négligeables). Ce projet 
est ainsi conçu d'un itinéraire extrêmement long, difficile à achever, mais résultant d'une profonde envie spirituelle dans mon cœur.

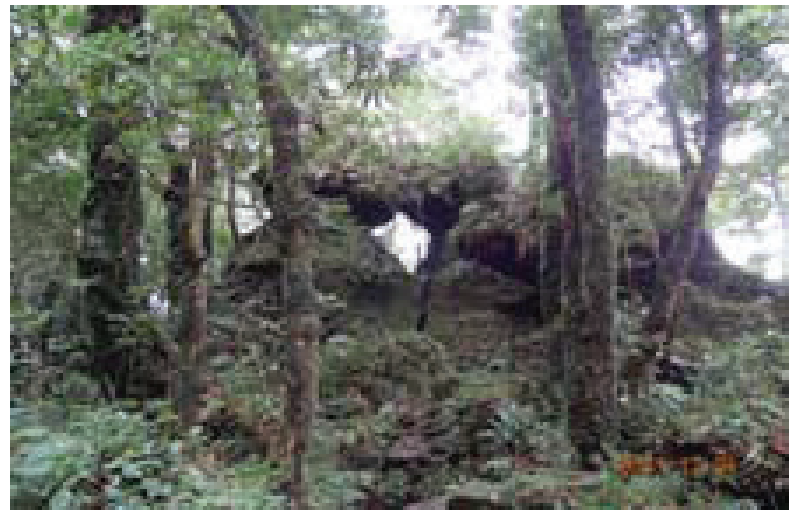

Figure 5 - Ruines de Ketangalan.

Sur le site principal de ce vestige, un trou se situe au milieu de la structure qui est un mythe au niveau du fonctionnement d'origine, mais qui s'inscrit parfaitement dans mon trajet d'iTunnel. Défini par moi-même en tant que dernier nœud de l'iTunnel, il ne signifie pas une fin, mais plutôt un portail vers le ciel, ou vers une histoire oubliée.

\section{Itinéraires et repères (tunnel et trous noirs)}

J'ai déterminé ensuite des trous de ver sur la carte. Pour en réaliser, j'imprime la carte en tant que maquette, je la plie plusieurs fois et puis la perce. En dépliant cette carte, plusieurs trous sont obtenus simultanément. Un tunnel se formera à partir de l'addition et la superposition de ces trous.
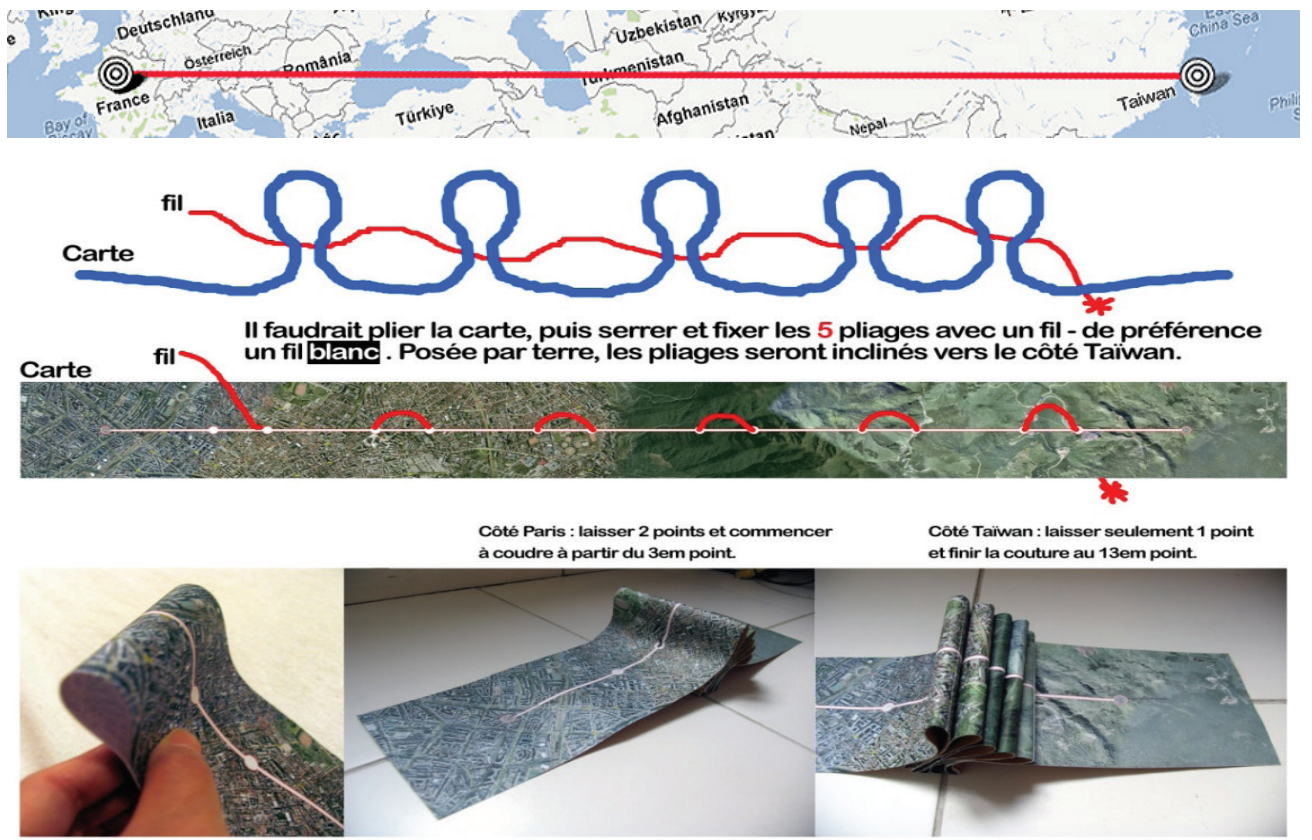

Figure 6 - Préparation des nœuds sur carte comme trajet, courtesy de l'artiste. 
Un trou sur la carte représente en réalité une zone d'à peu près 5 kilomètres de large. J'organise un voyage par zone, pendant lequel j'essaie de pénétrer (à l'aide de mon appareil photo), tous les bâtiments dans cette zone qui m'empêchent de poursuivre ma traversée.

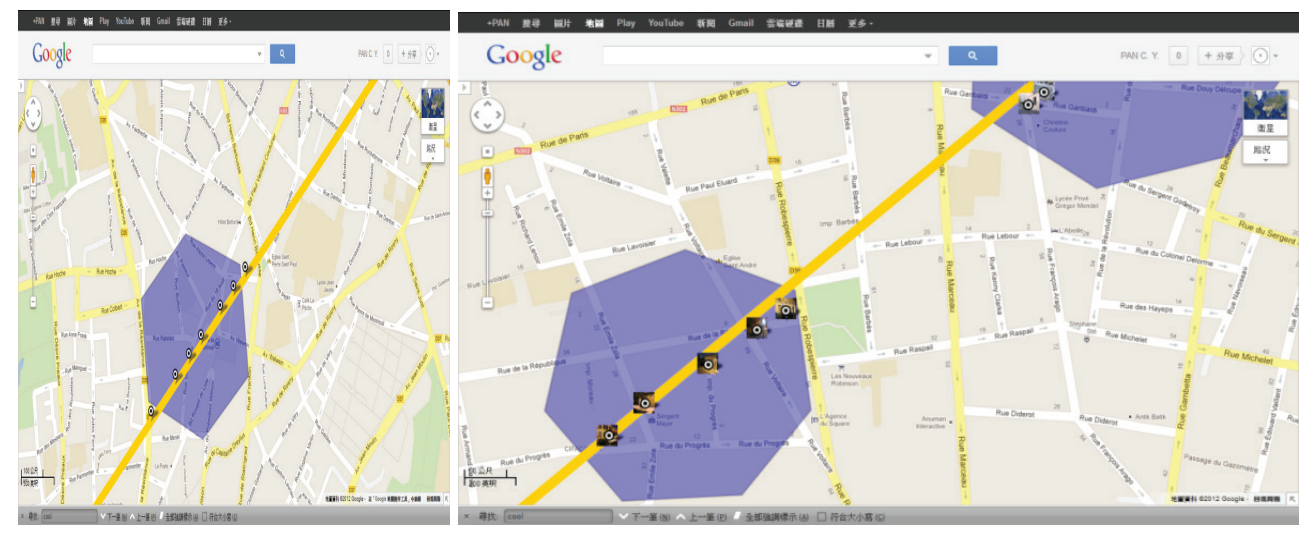

Figure 7 - Un trou sur la carte représente en réalité une zone (en bleu). À gauche: les points dans une zone représentent des trajets à réaliser. À droite : les trajets déjà réalisés dont ces photos sont téléchargées et intégrées dans ce plan. La grosse ligne en jaune foncé représente le iTunnel.

\section{L'action - les voyages}

Comme le trajet global s'avère extrêmement long $(8000 \mathrm{~km})$, plusieurs voyages se révèlent nécessaires pour en accomplir la totalité. De fait, il devient en réalité assez peu imaginable de réaliser tous les trajets. Cela ne me gêne guère, comptant plus sur la conception d'un travail artistique et moins sur la possibilité d'une réalisation. Jusqu'à présent, j'ai réalisé trois trajets dont vingt trous ont été achevés et capturés par photographies.

Le protocole de mes interventions se décline ainsi :

À chaque fois, je pars de chez moi pour débuter mes actions dans une zone à travailler. Quand la zone en question se révèle trop éloignée, j'emprunte les transports en commun : vélo, métro; puis train, lorsque les distances se révèleront trop importantes.

Durant les parcours dans la zone, je poursuis mon chemin toujours dans la même direction et au moment de faire face à un mur (bâtiment) qui empêche ma progression, je prends un cliché que je traiterai plus tard.

Les traces GPS de mon déplacement sont enregistrées sur mon téléphone portable et téléchargées en temps réel sur le site Web de l'exposition, sous la forme d'une ligne, un tracé qui se prolonge sur la carte, l'unique élément visible par le public présent dans la galerie à Limoges.

\section{Traitement d'images}

Après chaque retour, je traite les images en les inscrivant dans un dispositif spécifique, à savoir une forme ronde à l'origine d'un tunnel visuel et virtuel.

Sur le site Web, j'ai créé une interface interactive pour présenter à la fois le tunnel planifié, les endroits que j'ai parcourus, et les photographies que j'ai prises et qui ont 
été retouchées. En glissant la souris sur un des points de géolocalisation parcourus, la carte générale situera automatiquement ma localisation géographique d'origine, en affichant simultanément l'image, la photographie en question.

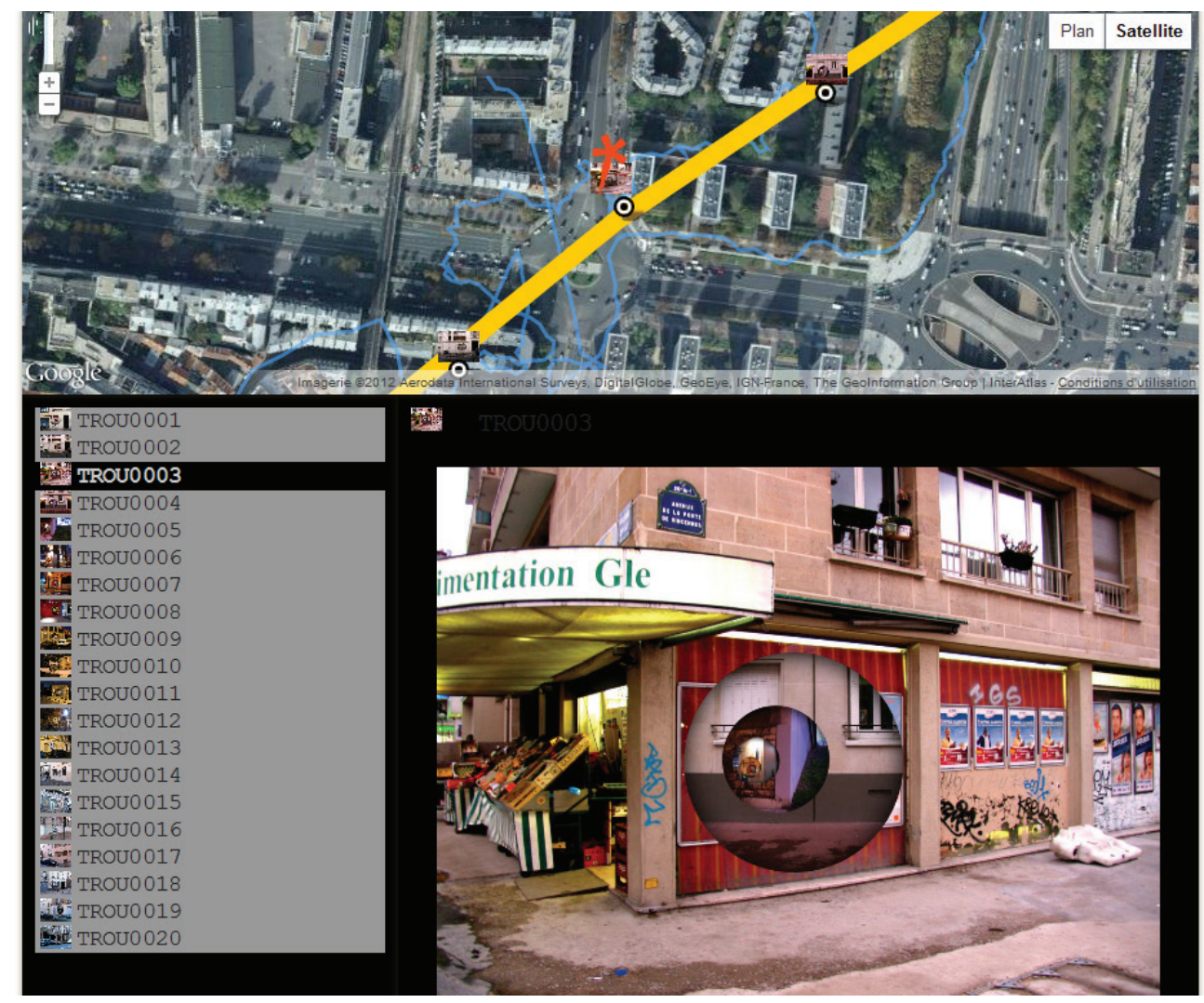

Figure 8 - Carte en ligne affichant à la fois le iTunnel planifié (ligne jaune), les traces que j'ai réellement parcourues (lignes bleus fines) et une liste des trous explorés accompagnés de ses repères géo-localisés sur la carte, ainsi que les images prises et retouchées.

\section{Rencontre à distance}

Chaque artiste dans cette exposition va effectuer une vidéoconférence avec le public dans la galerie. À mon tour, j'ai d'abord expliqué mon projet aux spectateurs et discuté avec eux. À la fin de la conférence, je partais afin de suivre mes trajets et les spectateurs découvrant sur l'écran mon déplacement sur la carte en temps réel, en goutant leur délicieuse soupe. On se trouve donc dans le cadre d'une action/ performance. 

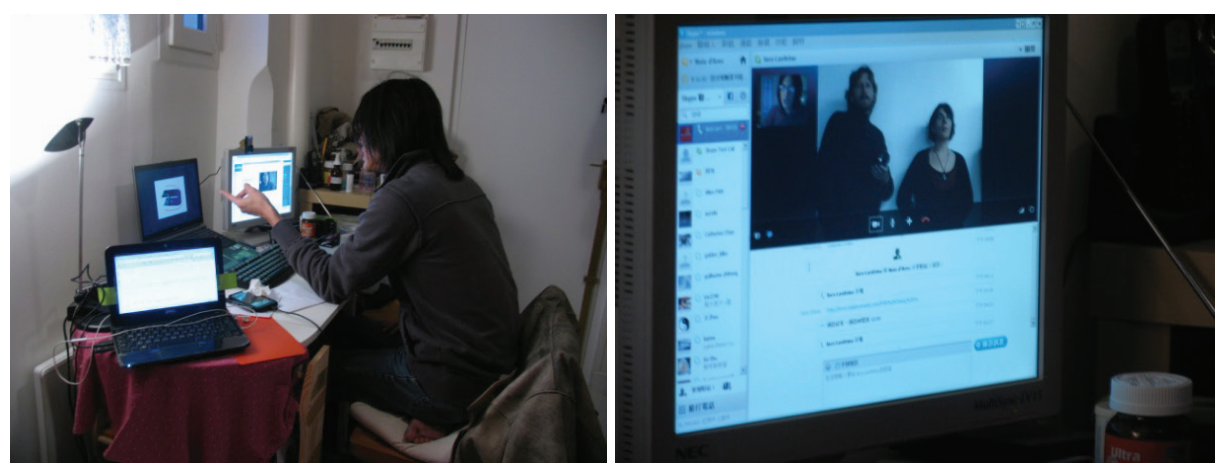

Figure 9 - Vidéoconférence avec le public via Skype, courtesy de l'artiste.

\section{Référence:}

McLuhan, M. Essential McLuhan. Eds. E. McLuhan and F. Zingrone, London: Routledge, 1995. 